\title{
A Comprehensive Study on a Novel Concentric Cylindrical Thermoelectric Power Generation System
}

\author{
Kuo Huang ${ }^{1}$, Bo Li ${ }^{1 *}$, Yuying Yan ${ }^{1,2 * *}$, Yong $\mathrm{Li}^{3}$, Ssennoga Twaha ${ }^{1}$, Jie Zhu ${ }^{1}$ \\ ${ }^{1}$ Fluids \& Thermal Engineering Research Group, Faculty of Engineering, University of Nottingham, UK \\ ${ }^{2}$ Fluids \& Thermal Engineering Research Centre, University of Nottingham Ningbo, China \\ ${ }^{3}$ School of Automotive and Mechanical Engineering, South China University of Technology, China
}

\begin{abstract}
This paper presents the novel designs of a concentric cylindrical thermoelectric generator (CCTEG) and an annular thermoelectric module (ATEM). The simulations are carried out to compare the performance of ATEM and the conventional square-shaped thermoelectric module (STEM). The heat pipe technology is introduced into the heat sink system in order to enhance the heat transfer in the radial direction of exhaust gas flow. A new index termed as the heat transfer filling factor $f$ has been introduced which quantities the level of space utilisation for thermoelectric modules (TEMs). The correlation between the coolant flow rate and TEM performance is also carried out. Experimental work is also carried out to demonstrate the viability of using the heat pipes for heat transfer enhancement as well proving the viability of the design. The simulations indicate that the open circuit electric potential of the ATEM is $17 \%$ more than that of the STEM. The experimental results show that the CCTEG system performs well under various conditions. This results also demonstrate that the concept of adding heat pipes to the heat sink system is a practical solution to achieve higher thermoelectric generator (TEG) performance while maintaining the compactness of the TEG system. A heat transfer filling factor of 0.655 is achieved for the CCTEG system which is higher compared to the existing TEG systems. Moreover, a higher coolant flow rate contributes to obtaining a better performance of the TEG system. It is important to note that the introduced index can give guidance for further optimisation design of TEG systems.
\end{abstract}

Keywords: Thermoelectric power generation, Heat enhancement, Exhaust heat, Temperaturedependent material properties, Heat pipes, Heat exchanger

\section{Introduction}

Previous decades have seen enormous developments in the renewable technologies by utilizing the alternative ways to obtain the usable energy. Thermoelectric heat recovery, as one of the most promising clean technologies, has attracted a greater attention due to its unique merits of energy conversion [1]. Unlike the traditional fossil fuel energy systems, TEG system converts waste heat directly into electricity through the Seebeck effect of the semiconductor materials whereby a temperature difference is maintained between the hot and cold side of the TEG module. TEG systems have the advantages of silent operation, high reliability, and they have no moving or mechanically complex components rendering them lasting for a very long time. They also operate at wider operational temperature range than most existing energy

\footnotetext{
* Corresponding author.

E-mail address: bo.li@ nottingham.ac.uk
} 
recovery systems $[2,3]$. However, the major challenge of the TEG system is the relatively low heat to electricity conversion efficiency[4]. Therefore, many research groups have made significant efforts on the improvement of the figure of merit for thermoelectric materials, such as $\mathrm{BiTe}, \mathrm{ZnBe}, \mathrm{SiGe}, \mathrm{TiO}$ and even new nanocrystal or nanowire thermoelectric materials. Therefore, due to such booming research, TEG systems will be possibly applied in automotive waste heat industry in the near future [5-7].

In addition to material research developments, thermal scientists and system engineers focus mainly on the optimisation of geometric parameter of TEMs and heat exchanger. Liu et al. have performed CFD simulation and experimental study on the structural design of the heat exchanger to improve the efficiency of TEG system [8]. Furthermore, the performance of the TEG system under real working conditions is examined and a maximum power output of 944 $\mathrm{W}$ is obtained [9]. Lee has studied the optimal design of thermoelectric devices with dimensional analysis [10]. The optimum designs including the power output and the efficiency on the external load resistance as well as the geometry of thermoelectric units. Liu et al. and Liang et al. have investigated the performance of two-stage thermoelectric generator systems $[11,12]$. The results show that the power output and conversion efficiency of the two-stage TEG increase significantly by changing the heat transfer coefficient of the two stages and are noted to be higher than those of the single-stage TEG.

The influence of the engine working condition and the boundary condition on TEG system performance in the vehicle are also being researched. Yu et al. found out that multiple vehicular driving conditions might lead to the significant variation of TEG performance [13]. The results indicate that the engine speed is a major factor affecting the TEG performance. The results deduce that the higher the vehicle speed, the better the TEG performance. A higher road grade is also spotted to increase the power output of TEG significantly. He et al. have studied the influence of different cooling methods on the performance of TEG system [14]. The results reveals that the power output is higher with liquid cooling than air cooling whereas the counterflow arrangement leads to higher power output though a much larger area is needed compared to the parallel-flow method. Other factor which needs consideration are the space and weight of the vehicle. It is noted that, in practice, there is limited space in the vehicle exhaust system; so the light weight and the size of the TEG are also vital factors in applying the TEG for fuel economy [15]. Wang et al. presented a TEG design with heat pipes-assisted heat exchanger structure to overcome the common conflicts between the needs for high heat flux into TEG and the limitation of overall heat exchanger dimensions and weights. Therefore, the need of light weighted thermoelectric generator system is very necessary for the development of vehicular TEG systems. The existing TEG systems are often too bulky to be applied in the exhaust system.

In this paper, a novel design of concentric cylindrical thermoelectric generator (CCTEG) system is presented for use in the exhaust system in the passenger car. The new design is capable of adapting to the shape of the exhaust pipe besides accommodating more TEMs unlike other TEG system designs of the same installation length. Given the form of the CCTEG system, a new annular thermoelectric module (ATEM), which is different from the previous square thermoelectric module (STEM), is also designed for the use of CCTEG system. Therefore, a relatively compact heat exchanger is necessary for the TEG system. In addition, 
heat pipes are applied to enhance the radial heat transfer of the whole system by means of conducting the heat directly into the annular TEM modules [16, 17]. Mathematical analysis for the design are presented and experiments are carried out to verify the feasibility of CCTEG. Simulations have been done to investigate the difference in performance between the annular TEM and square TEM. Moreover, different performance indicators of evaluation are used to compare these two different shapes of TEMs and analyse the geometric influence on the TEM. Additionally, a model-scale experimental prototype and test rig for CCTEG are also constructed to examine the feasibility of using of heat pipes in this TEG system.

\section{The Design of Concentric Cylindrical Thermoelectric Generator}

The described concentric cylindrical TEG design is as shown in Fig. 1. The main components of this TEG are the hot plates, cooling plates, heat pipes, concentric thermoelectric modules, and water jacket. The arrows indicated that the waste heat of the exhaust gas is conducted by heat pipes towards the hot side of the thermoelectric generator. On the cold side of TEM, the cooling plate also discharges heat to the water jacket via heat pipes. The purpose of using heat pipes is to divert the waste heat in the radial direction from engine exhaust pipe and discharge the heat to the water jacket. In addition, the heat transfer in the radial direction is enhanced due

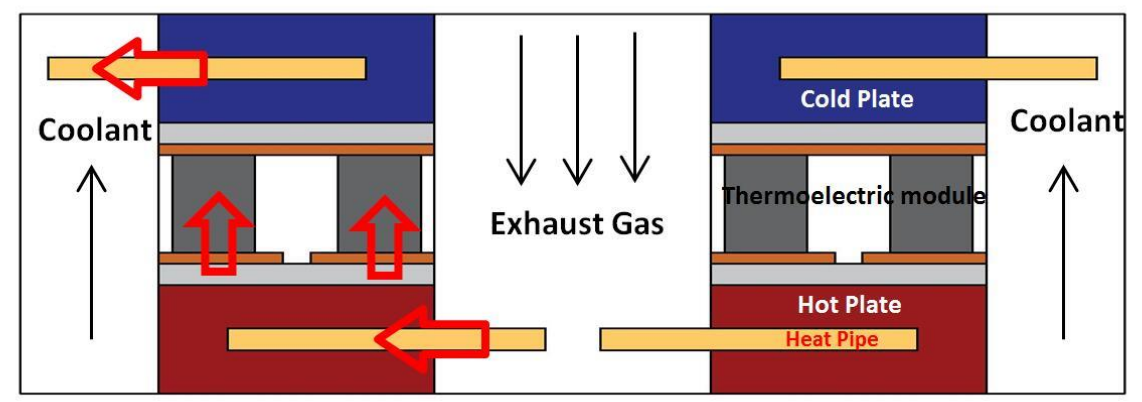

Fig. 1. Schematic of the concentric cylindrical thermoelectric generator

to the characteristic of super heat conductivity of the heat pipes.

The competitive advantage of CCTEG is the less installation space and weight required than conventional bulky TEG system. Since the installation space in vehicle exhaust system is limited in most scenarios, a relatively compact heat exchanger is imperative for the implementation of TEG systems. Moreover, the weight of the overall auxiliary devices is also a key factor for the fuel consumption of vehicles. The application of heat pipes makes it possible to reduce the weight of heat exchanger, rendering the CCTEG lighter than any other TEG systems. 


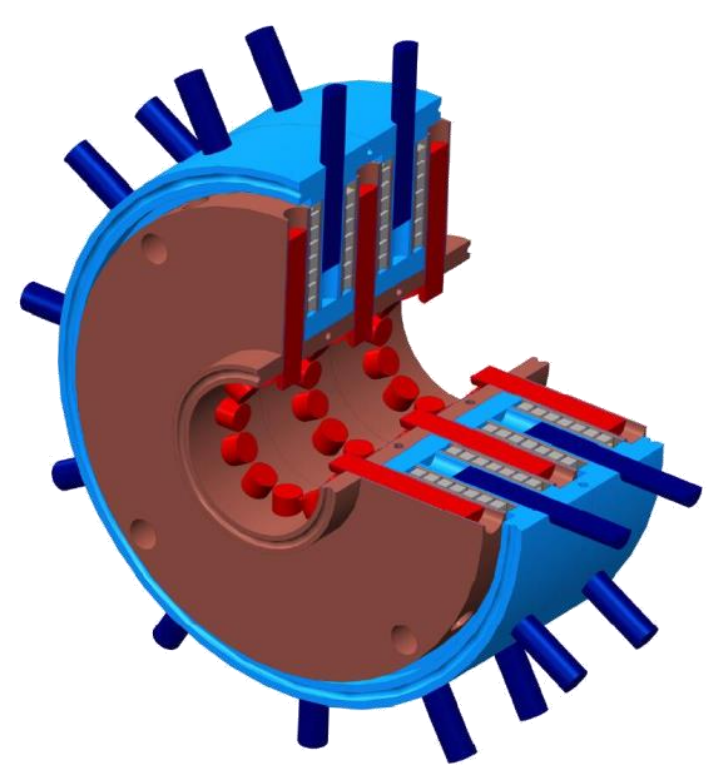

a

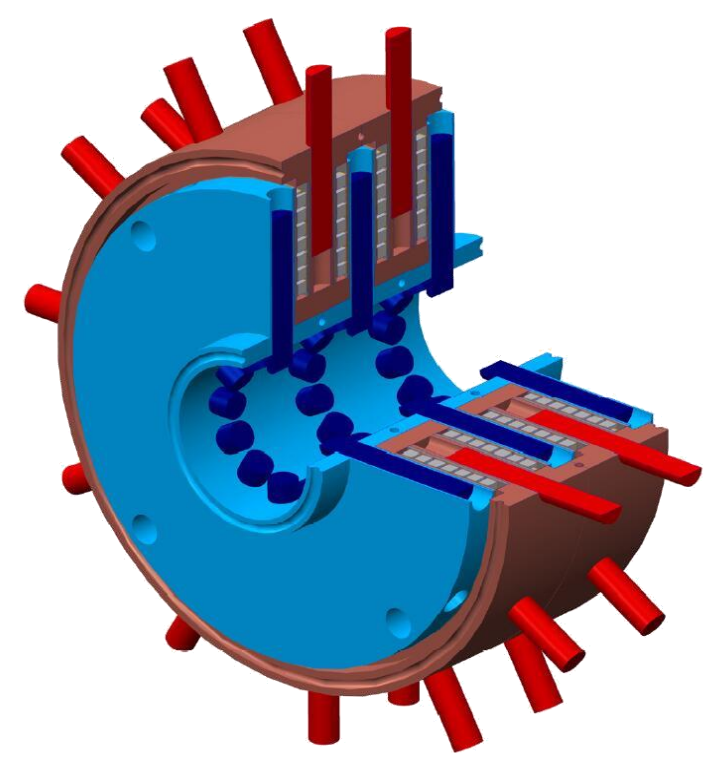

$\mathbf{b}$

Fig. 2. 3D schematic of the concentric cylindrical thermoelectric generator

(a) Exhaust gas flow through inner pipe (b) Exhaust gas flow through outer pipe

As depicted in Fig. 2, the two sections represent the minimum repeat units of the overall system which can be segregated by the need of power rating. The minimum repeat unit is comprised of 4 ATEMs, 3 hot plates and 2 cooling plates including 12 heat pipes in each plate marked in blue and red colour in the Fig. 2 (a) and 2(b) respectively. The exhaust gas flows throw the inner pipe of the system in Fig. 2 (a) while the coolant flows in a constrained space shaped by the heat pipes, the surface of the cooling plate and the shell of the heat exchanger. Considering the various applications and the performance needs, this configuration can be interchanged by switching the channels of the gas and the coolant flows. As described in Fig 2 (b), the coolant flow can be arranged in an axial flow by replacing the gas flow in Fig.2 (a) baring mind the heat convection in the coolant. The gas flow, therefore, can be allocated at the outer side where the extra heat sinks on the heat pipes can be installed. In this way, a higher power generation performance can be achieved due to the heat transfer enhancement between the heat pipes and gas flow.

\section{The Modelling of Concentric Cylindrical Thermoelectric Generator}

The three-dimensional models of STEM and ATEM are analysed in CAD software. Both thermal analysis and thermoelectric performance of these two models are simulated in the CFD software.

\subsection{Material properties of TEM for simulation and experiment}

The previous studies of the thermoelectric generator mainly focused on the heat exchanger and the improvement of thermoelectric materials, but the influence of the topological shape of thermoelectric modules has not attracted much attention by the researchers. A different design 
for the thermoelectric module - ATEM is described in this paper. The aim of this module is to fit the novel design of CCTEG system.

The two different shapes of thermoelectric modules that are described in this study are shown in the Fig. 3. Both modules share the same number of thermoelectric units and the same material properties. The geometric details of the TEMs are listed in Table 1.

Table 1 Geometric details of TEMs

\begin{tabular}{|c|c|c|c|c|}
\hline \multirow{2}{*}{ Parameters } & \multirow{2}{*}{ Thermoelectric Leg } & \multirow{2}{*}{ Copper } & \multicolumn{2}{|c|}{ Ceramic Plate } \\
\hline & & & Square TEM & Annular TEM \\
\hline Length (mm) & 2.55 & 5.8 & 65.7 & - \\
\hline Width (mm) & 2.55 & 2.55 & 65.7 & - \\
\hline Height (mm) & 2.55 & 0.3 & 0.7 & 0.7 \\
\hline Inner Diameter (mm) & - & - & - & 50.8 \\
\hline External Diameter (mm) & - & - & - & 101.6 \\
\hline
\end{tabular}

Each TEM in this study contains 210 units. These units are electrically connected in series implying that the electrical currents through them are the same. On the other hand, the heat passes through these units simultaneously, meaning that they are thermally connected in parallel. A single TEM unit includes a P-type and an N-type leg, connected together with copper strips. The electrical insulation of the TEM units is achieved by placing AIN ceramic plate on the top and bottom sides of modules.

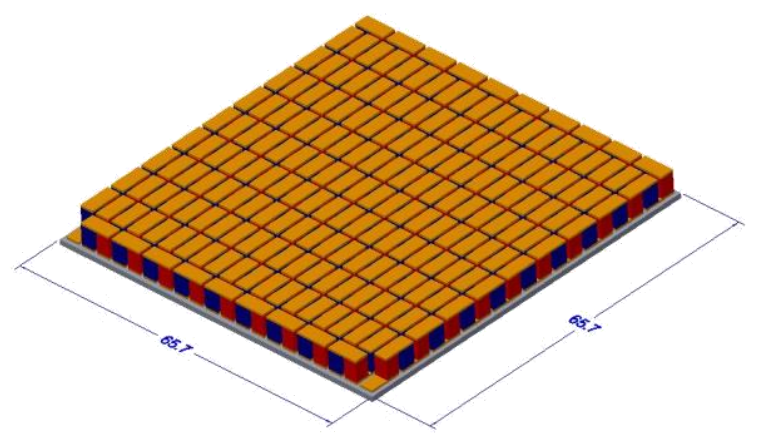

(a)

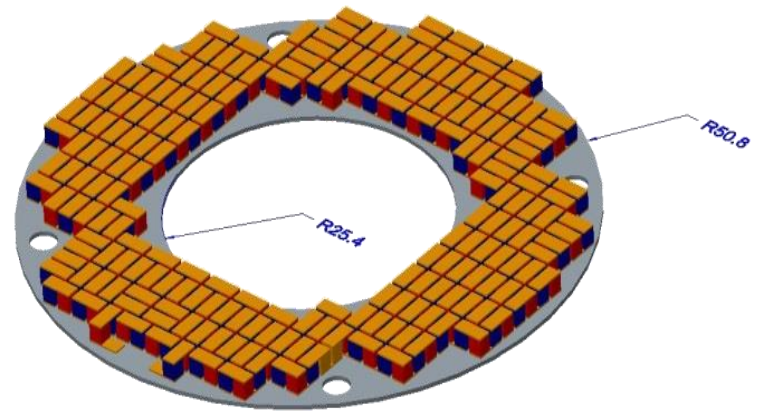

(b)

Fig. 3. Structure of (a) square TEM and (b) annular TEM

During the simulation, the temperature-dependent Seebeck coefficient, thermal conductivity, and the electrical resistivity for both $\mathrm{N}$-type and P-type thermoelectric legs are given by the polynomials whose coefficients are summarized in Table 2 [18-20]. The coefficients of polynomials represent the material properties from which the TEG modules are made. 
Table 2 Material parameters of a single TEM unit

\begin{tabular}{|c|c|c|c|c|c|c|c|c|}
\hline Parameters & & & Semico & ductors & & & Copper & $\begin{array}{c}\text { ALN } \\
\text { Ceramic }\end{array}$ \\
\hline \multirow{3}{*}{$\begin{array}{c}\text { Seebeck } \\
\text { coefficient } \\
(\mathbf{V} / \mathbf{K})\end{array}$} & & $\alpha_{0}\left(10^{0}\right)$ & $\alpha_{1}\left(10^{-7}\right)$ & $\alpha_{2}\left(10^{-9}\right)$ & $\begin{array}{c}\alpha_{3}\left(10^{-}\right. \\
12)\end{array}$ & $\begin{array}{c}\alpha_{4}\left(10^{-}\right. \\
16)\end{array}$ & \multirow{3}{*}{-} & \multirow{3}{*}{-} \\
\hline & P-type & 0.0002 & 7 & -3 & 4 & -5 & & \\
\hline & N-type & -0.0002 & -3 & 1 & -1 & 1 & & \\
\hline \multirow{3}{*}{$\begin{array}{c}\text { Thermal } \\
\text { conductivity } \\
(\mathbf{W} / \mathbf{m} \cdot \mathbf{K})\end{array}$} & & $\kappa_{0}\left(10^{0}\right)$ & $\kappa_{1}\left(10^{-5}\right)$ & $\kappa_{2}\left(10^{-7}\right)$ & $\begin{array}{c}\kappa_{3}\left(10^{-}\right. \\
10)\end{array}$ & $\kappa_{4}\left(10^{-12}\right)$ & \multirow{3}{*}{350} & \multirow{3}{*}{285} \\
\hline & P-type & 0.016 & -4 & 2 & -6 & 3 & & \\
\hline & N-type & 0.0122 & -3 & 6 & 3 & -8 & & \\
\hline \multirow{3}{*}{$\begin{array}{c}\text { Electrical } \\
\text { conductivity } \\
(\mathbf{S} / \mathbf{m})\end{array}$} & & $\rho_{0}\left(10^{0}\right)$ & $\rho_{1}\left(10^{-6}\right)$ & $\rho_{2}\left(10^{-9}\right)$ & & & \multirow{3}{*}{5.90} & \multirow{3}{*}{ - } \\
\hline & P-type & 0.0007 & 7 & -1 & & & & \\
\hline & N-type & 0.0009 & 6 & -9 & & & & \\
\hline
\end{tabular}

\subsection{Simulation boundary conditions}

The thermoelectric material used in the simulation is bismuth telluride $\left(\mathrm{Bi}_{2} \mathrm{Te}_{3}\right)$. The numerical simulation is performed at a steady state for both of the two modules. The governing equations for the electric and conjugate heat transfer are modified according to the conditions set out to perform the analysis of the designs.

The heat flux and heat flow equations are

$$
\begin{gathered}
q=\alpha T J-\kappa \nabla T \\
\dot{Q}=q \cdot A
\end{gathered}
$$

Thermoelectric effects are caused by coupling between charge transport and heat transfer. Under isotropic conditions, the linear relations are presented as

$$
\begin{gathered}
E=J \rho+\alpha \nabla T \\
J=\sigma(E-\alpha \nabla T)
\end{gathered}
$$

where $\sigma=1 / \rho$ is the electric conductivity; $E$ is the electric field; $\nabla T$ is the temperature gradient. The electric field of the thermoelectric material is affected by the current density and the temperature gradient. The coefficients are known according to the Ohm's law and the Seebeck effect. These two equations are based on Onsager-Callen theory on irreversible thermodynamics [21].

As for the steady-state conditions, the governing equations for constant current and heat transfer of inhomogeneous thermoelectric material can be described by

$$
\begin{gathered}
\nabla \cdot J=0 \\
\nabla \cdot(\kappa \nabla T)+J^{2} \rho-T J\left[\left(\frac{d \alpha}{d T}\right) \cdot \nabla T+\left.\nabla \alpha\right|_{T}\right]=0
\end{gathered}
$$

where $\left.\nabla \alpha\right|_{T}$ is the gradient at a given temperature.

Considering one of the thermoelectric units to represent the whole CCTEG module, assuming a uniform material and dimensions for each of the units, Eq. (8) for one-dimensional analysis at steady state gives the non-linear differential governing equation as; 


$$
\frac{d^{2} T}{d x^{2}}-\frac{I \frac{d \alpha}{d T}}{A \kappa} T \frac{d T}{d x}+\frac{I^{2} \rho}{A^{2} \kappa}=0,\left(T_{(0)}=T_{c}, T_{(L)}=T_{h}\right)
$$

where $A$ is the cross-sectional area of the thermoelectric units [22].

In practice, the TEG system absorbs the heat from the engine exhaust gas and uses a coolant to obtain a higher temperature gradient between the cold and hot side. Therefore, the heat transfer coefficient is the key factor which influences the heat transfer in the TEM. The heat transfer coefficient for the forced air convection and forced water convection, in the present study, are set at $40 \mathrm{~W} / \mathrm{m}^{2} \cdot \mathrm{K}$ and $6000 \mathrm{~W} / \mathrm{m}^{2} \cdot \mathrm{K}$ respectively.

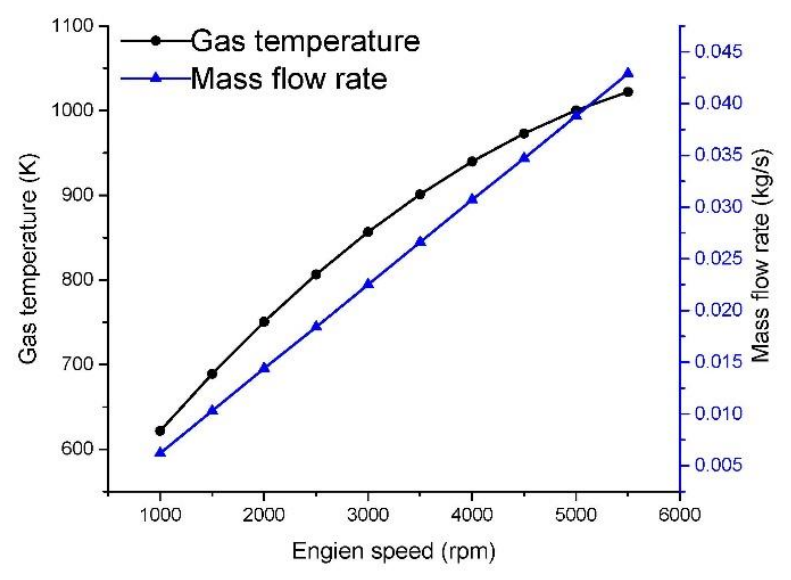

Fig. 4. Boundary conditions for the performance simulation

The capacity of the TEG system is highly related to the mass flow rate and the temperature of exhaust gas, which is closely associated with the engine speed and the vehicle load. The boundary conditions used in the simulation are shown in Fig. 4 [15]. During the simulation, the hot side boundary condition of a single TEM is the inward heat flux or hot side temperature which is calculated by the exhaust gas temperature and mass flow rate. This is intended to ensure that the temperature maintained at the same value under the same engine speed. Moreover, the heat transfer coefficients on the hot and cold side of both TEM remain unchanged.

\subsection{Description of the experimental setup}

A scaled-up experimental prototype of CCETG was configured and measurements of its overall performance were carried out under various boundary conditions. The schematic of the test rig is shown in Fig. 5 and Fig. 6. The TEG block was composed of 16 thermoelectric modules in four layers, with cooling plates and hot plates. The TEMs were made of a semiconductor material $\mathrm{Bi}_{2} \mathrm{Te}_{3}$ and had dimensions of $40 \mathrm{~mm}$ x $40 \mathrm{~mm}$ x $3.8 \mathrm{~mm}$ composed of 210 thermoelectric units. Thermal interface materials were placed at the interface of solid surfaces to enhance the heat transfer. 
Two controllable cartridge heaters rated at $100 \mathrm{~W}$ were used to supply heat on the hot side. The heat supply to the TEG system could be adjusted by changing the voltage input. This system was cooled by water through the water jacket. The temperature at the heat source and

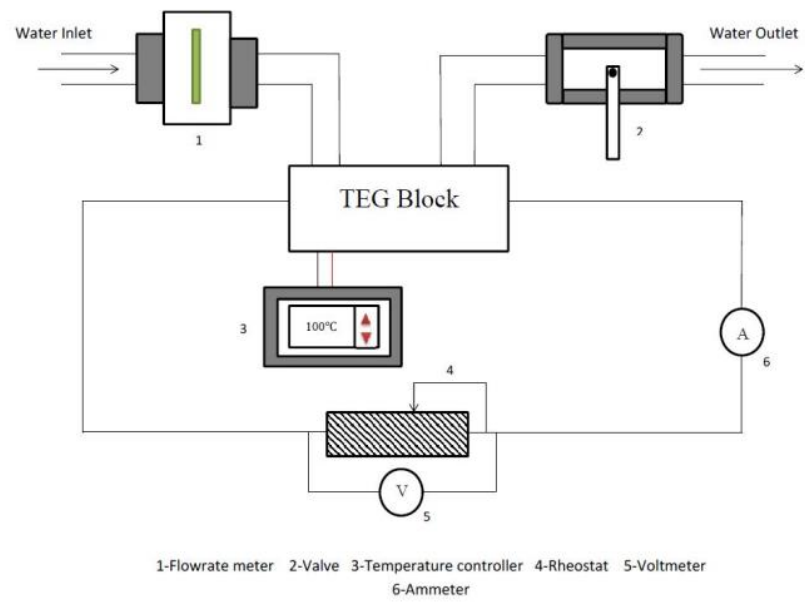

Fig. 5. Schematic of the test rig

heat sink was measured by K-type thermocouples and the flow rate of cooling water was measured by a flowmeter. The thermocouples were located in slots drilled on the surface of the hot plate. The temperature, flow rate and voltage were recorded by a DT500 series III Data taker. A rheostat resistance ranging from $0 \Omega$ to $40 \Omega$ was connected to the TEG system to provide the adjustable external load.

However, the result of the experimental test shows some discrepancy with the expected results, which is caused by the instrumental errors. The difference between the expected and actual maximum power obtained is dependent on the errors in both voltage and current measurements. The error in the heat flux temperature set-point was based on the thermocouple

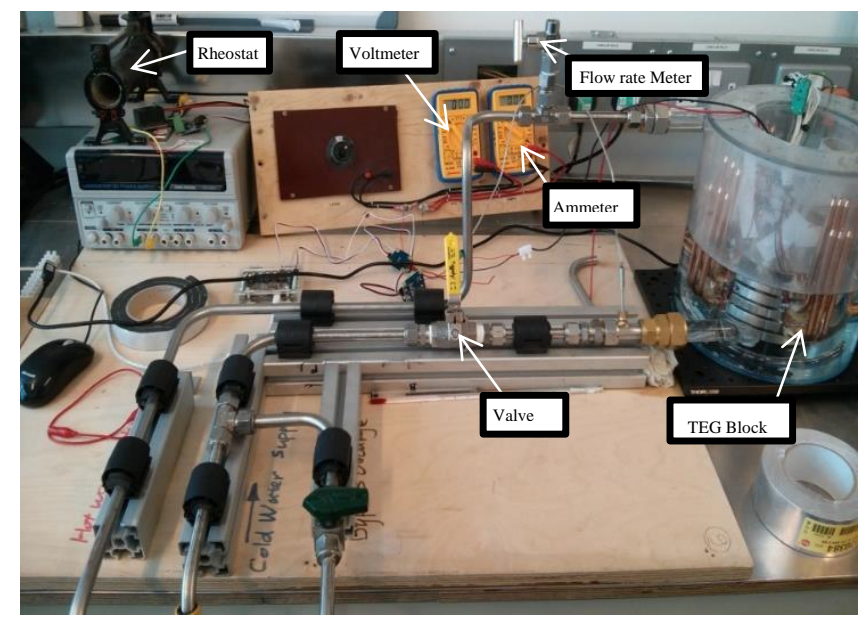

Fig. 6. Image of the constructed test-rig utilised throughout the experiment

accuracy and the errors in the temperature controller. The errors in the temperature controller were a combination of the indication error of the thermocouple temperature and the overshoot deviation from the set-point temperature. 


\section{Results and Discussion}

\subsection{Performance comparisons of single TEM}

Assuming that the inward heat flux of each TEM remains constant, the total inward heat flow is proportional to the heat transfer area according to Eq. (5) and (6). Based on the geometric comparisons in Table 1, the main difference between these two TEMs is the heat transfer area. The available heat transfer areas for the STEM and ATEM are found to be $4316.49 \mathrm{~mm}^{2}$ and $6077.41 \mathrm{~mm}^{2}$ respectively through calculations.

According to energy conservation, the power output generated by a single thermoelectric unit is expressed as:

$$
P_{\text {out }}=Q_{h}-Q_{c}=\alpha I\left(T_{h}-T_{c}\right)-I^{2} R
$$

The power generated at the load is expressed as:

$$
W=I^{2} R_{L}
$$

where $R_{L}$ is the load resistance.

Given the open circuit electric potential $V=\alpha \cdot \nabla T$, the current in circuit is expressed as [23]:

$$
I=\frac{\alpha\left(T_{1}-T_{2}\right)}{\left(R_{L}+R\right)}
$$

The maximum power efficiency is the specific conversion efficiency where the power reaches the maximum. From Eq. (14) and (15), the maximum power can be computed as:

$$
\dot{W}=\left(\frac{\alpha \Delta T}{\frac{R_{L}}{R}+1}\right)^{2} \frac{R_{L}}{R}
$$

The maximum power is obtained at $\mathrm{R}_{L}=\mathrm{R}$ and by inserting this relation into Eq. (16), this gives:

$$
\dot{W}_{\text {max }}=\frac{\alpha^{2} \Delta T^{2}}{4 R}
$$

The conversion efficiency of the TEG is in the form of [24]

$$
\eta_{t}=\frac{W}{Q_{h}}
$$

Fig. 7 (a) and (b) show the different performances of each TEM, under the same inward heat flux at $5 \mathrm{~W} / \mathrm{cm}^{2}$. From the results, the heat transfer area has a considerable level of influence on the temperature distribution on the hot side of the TEM. Since the heat exchange area of ATEM is larger than that of STEM, ATEM can conduct more heat with the same inward heat flux. In this case a higher temperature gradient between the hot and cold side of TEM for the ATEM resulting into a higher open circuit electric potential. Fig. 7 (a) and (b) indicate that the average hot side temperature of ATEM is $602 \mathrm{~K}$ which is higher than the average hot side temperature of the STEM which is $500 \mathrm{~K}$. The temperature gradient between both sides of ATEM and STEM is $213 \mathrm{~K}$ and $150 \mathrm{~K}$ respectively. As a result, the ATEM generates a $14.5 \mathrm{~V}$ of open circuit electric potential which is $2.1 \mathrm{~V}$ higher than that of STEM under the same heat flux condition. 


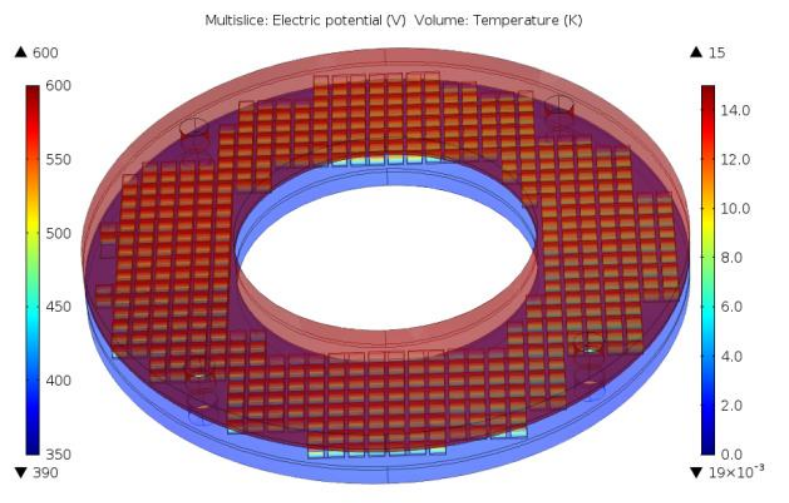

(a)
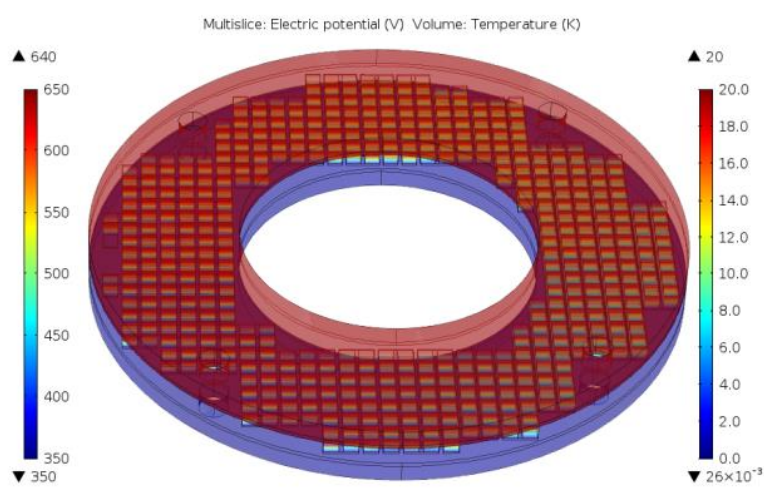

(c)

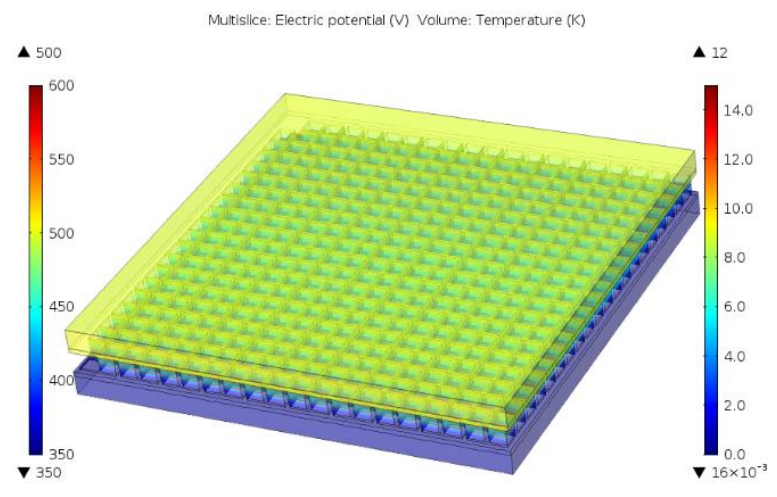

(b)

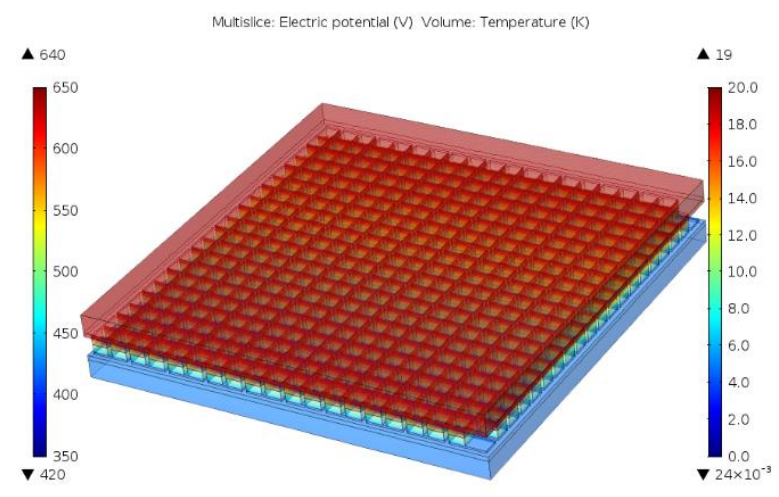

(d)

Fig. 7. Performance comparison for ATEM (a \& c) and STEM (b \& d)

1. (a \& b) under inward heat flux $q=50000 \mathrm{~W} / \mathrm{m}^{2}$ (Neumann boundary condition), heat transfer coefficient $h_{c}=6000 \mathrm{~W} / \mathrm{m}^{2} \mathrm{~K}$, $T_{\text {coolant }}=288 \mathrm{~K}$ (Cauchy boundary condition on the cold side $)$

2. (c \& d) at hot side temperature $T_{h}=643 \mathrm{~K}$ (Dirichlet boundary condition), heat transfer coefficient $h_{c}=6000 \mathrm{~W} / \mathrm{m}^{2} \mathrm{~K}, T_{\text {coolant }}=$ $288 K$ (Cauchy boundary condition on the cold side)

Fig. 7 (c) and (d) show the simulation result of both TEMs operating at the same hot side temperature of $643 \mathrm{~K}$. The figures indicate that the ATEM module performs better than STEM at the same hot side temperature. As long as the hot side temperature is kept the same for the two TEMs, a lower average cold side temperature for ATEM is achieved due to the larger heat transfer area enabling it to acquire a higher heat dissipation rate. Consequently, the respective average cold side temperatures for ATEM and STEM are $352 \mathrm{~K}$ and $417 \mathrm{~K}$, representing a difference of $65 \mathrm{~K}$ in the two TEMs. This difference obviously affects the open circuit electric potential generated by TEM because the Seebeck effect responsible for generating this potential is actuated by the temperature difference on the two sides of the TEM. Therefore, the open circuit electric potential for ATEM is higher at 19.6 V than the one for STEM at $18.9 \mathrm{~V}$.

As mentioned above, two kinds of boundary conditions are adopted. The first one is Neumann boundary condition on the hot side and Cauchy boundary condition on the cold side. The second one is Dirichlet boundary condition on the hot side and Cauchy boundary condition on the cold side. The reason for specifying these two different sets of boundary conditions is that the various measurements hot side temperature in practical and simulation are taken. In some situation, the exact value of hot side temperature is difficult to be obtained, so the temperature is often calculated by the heat flux on the hot side which is the Neumann boundary 
condition. During simulation, the hot side temperature is often specified for a more accurate simulation. As a result, Dirichlet boundary condition is also applied in the second part of the simulation.

\subsection{Influence of heat transfer area}

Under the same inward heat flux, the primary difference of these two TEMs is the heat transfer area which leads to hotter surface temperature distribution at ATEM than at STEM hot side surfaces. As a result, the different temperature distributions of the two TEMs influence their power generation capabilities represented by their open circuit electric potential values. In this part of the study, the influence of engine speed is taken into consideration, as the variation of engine speed affects the total inward heat flux of TEM. In this way, the influence of automotive driving cycle to the performance of TEM is investigated.

Fig. 8 shows the average temperature of both TEMs on the hot side under different inward heat flux which is calculated at various engine speeds. The curves indicate that the hot side average temperature of ATEM is higher than that of STEM due to the $28.9 \%$ larger heat transfer area of ATEM. Furthermore, the hot side average temperature is in direct proportion to inward heat flux. Under the same inward heat flux, the hot side average temperature of ATEM is 20 $\sim 25 \mathrm{~K}$ higher than that of STEM. This difference results in a considerable variation in the temperature gradient so that the open circuit electric potential of ATEM is again greater than that of STEM.

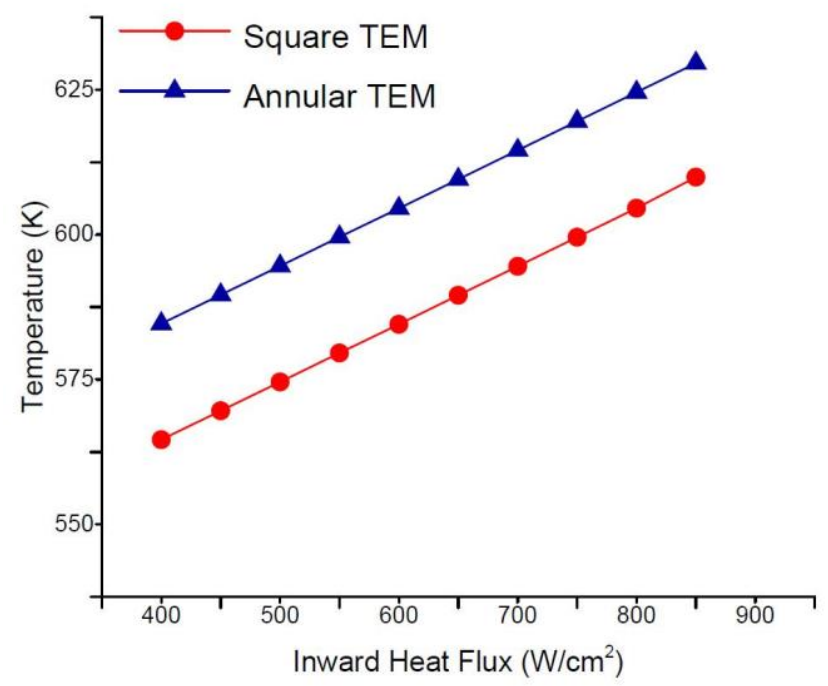

Fig. 8. Hot side average temperature of both TEM under different inward heat flux

Fig. 9 shows the open circuit electric potential of the two TEMs under different engine working conditions. Since the temperature gradient of ATEM is higher than that of STEM, the open circuit electric potential generated by ATEM is also higher in general. Under various inward heat fluxes caused by different engine speed, the open circuit voltage generated by ATEM is $2.5 \mathrm{~V} \sim 2 \mathrm{~V}$ higher than STEM. This difference becomes larger when the inward heat flux is lower because the two curves show a parabolic shape. This is due to the characteristic of semiconductor material which is considered as temperature-dependent in this case, which is different from previous investigations with constant material parameters [25]. 
The study also demonstrates that each TEM has an optimal performance point which is a function of inward heat flux as observed in Fig. 9. Therefore, the automotive driving condition should be taken into account when the TEG system is being designed for a specific vehicle exhaust system. In order to obtain a better output voltage and power, the position of TEG system in the entire exhaust system also needs to be considered carefully.

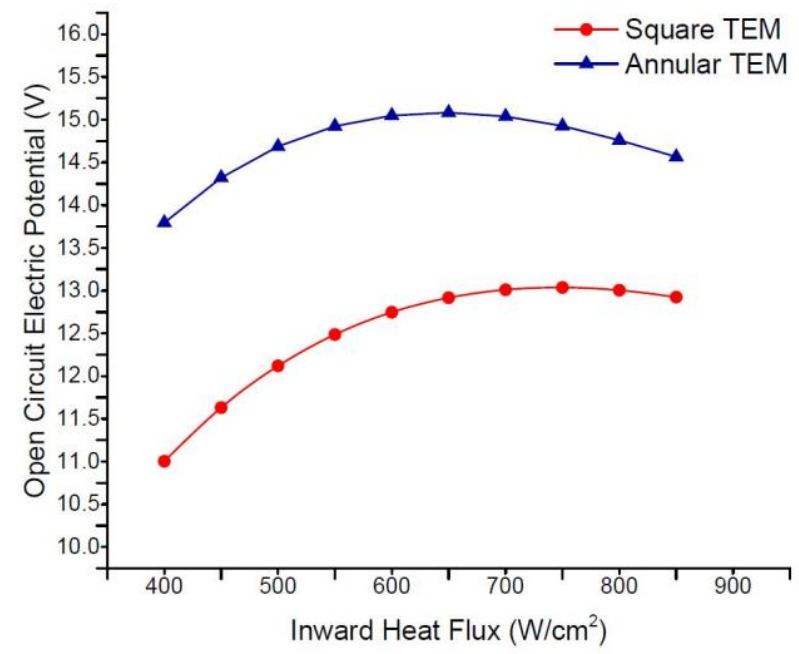

Fig. 9. Open circuit electric potential of both TEM under different inward heat flux

\subsection{Experimental results}

In addition to the simulation studies, proof-of- concept test is also carried out in a model-scale test rig and results are compared with those of the simulations. In addition, to verify the accuracy of these results, Hi-Z performance calculator is utilised during the comparative analysis of the experimental and simulation results.

Fig. 10 shows the comparison of the variation in power output versus varying load resistance $\left(R_{L}\right)$ under different hot side temperature. As shown in Fig. 10, three load curves exhibit the same trends though they have a marginal difference under different load resistance conditions. The curves of simulation and Hi-Z calculator in Fig. 10 (a), 10 (b) and 10 (c) have a similar at a lower load resistance but slightly deviate from each other at a higher load resistance. These differences could be caused by the difference in accuracies and errors during calculations of the internal electric resistance and the heat flux. In Hi-Z performance calculator, there is an egg crate area which has a lower heat transfer coefficient than other areas. Besides, the internal electric resistance in the simulation is calculated by continuous integration method which is different from Hi-Z performance calculator. In addition, the application of different input heating power may also cause the difference between these curves. 


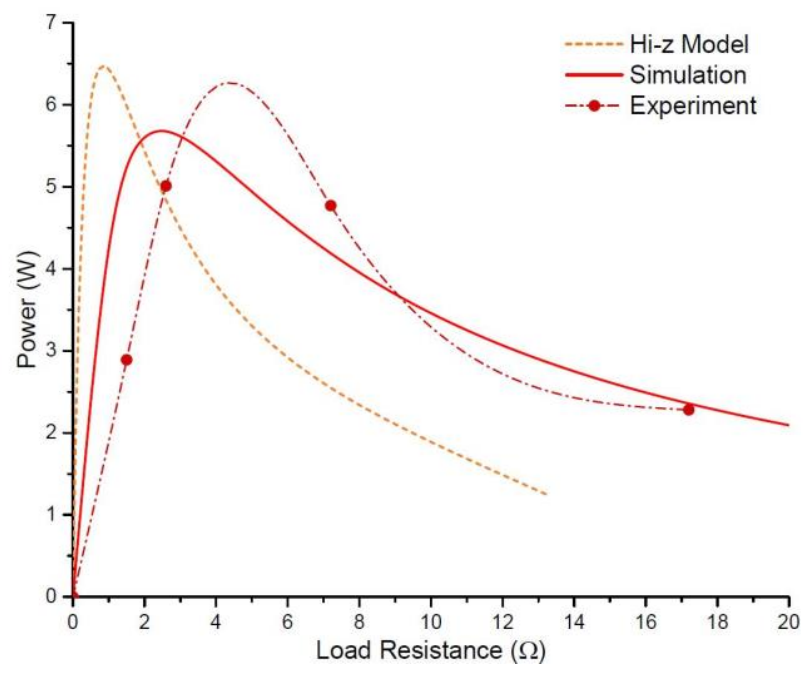

(a)

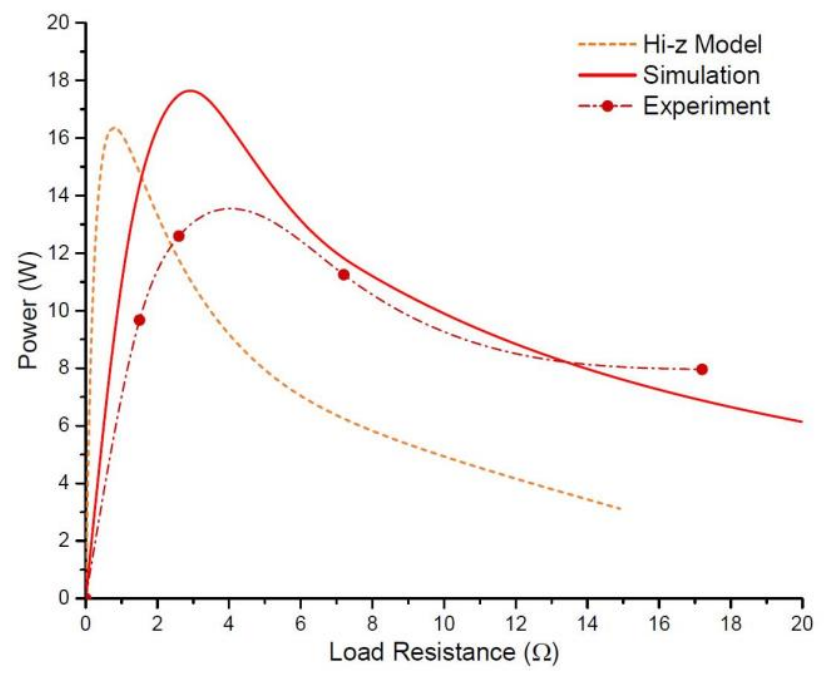

(b)

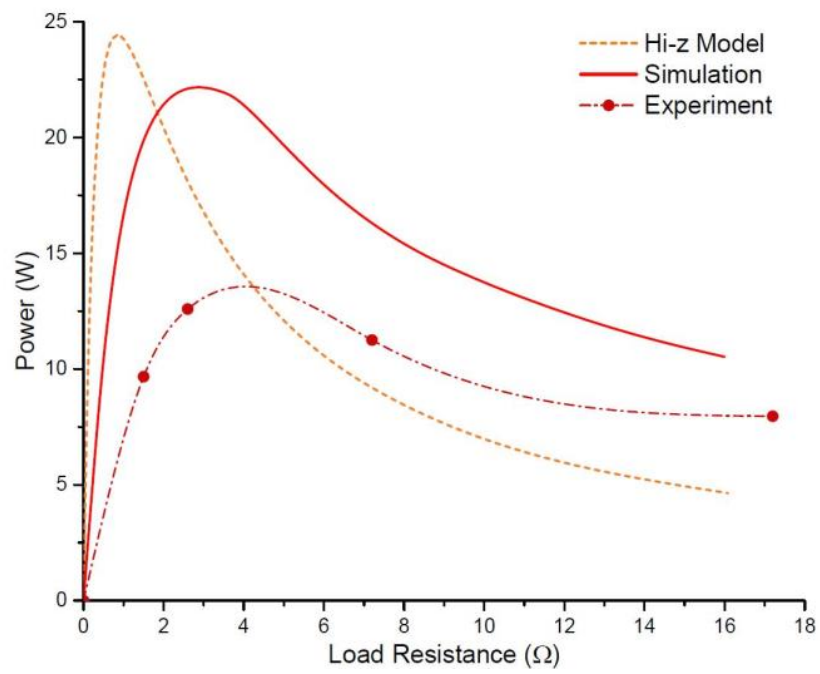

(c)

Fig. 10. Comparison of model performance predicted by the simulation, Hi-Z calculator, and experiment result

(a) under $353 \mathrm{~K}$ of the hot side temperature; (b) under $393 \mathrm{~K}$ of the hot side temperature; 
(c) under $423 \mathrm{~K}$ of the hot side temperature;

The trend of these three power output curves is practically the same although different hot side temperature is applied. The peak point of each curve occurs when the inner resistance of the TEM matches the external load resistance. The power output increases sharply at lower load resistance and declines slowly at a higher load resistance which seem to be relatively levelling off afterwards. In Hi-Z Model, the increment and reduction of the power output are more noticeable due to the differences in the calculation method. The agreement between the simulation results and the experimental data is better than that of Hi-Z Model.

Fig. 10 (a) is the comparison of the simulation, Hi-Z calculator and experiment results at 353 $\mathrm{K}$ for the hot side temperature, using the same thermal conductivity obtained in the previous part. The hot side temperature is as a result of different inward heat flux which is used in simulation and varies from $353 \mathrm{~K}$ to $384 \mathrm{~K}$ representing an increment of $31 \mathrm{~K}$. The comparisons of the different hot side temperatures are shown in Fig. 10 (b) and (c). These results show good agreement with the result at $353 \mathrm{~K}$ hot side temperature in Fig.10 (a). When the hot side temperature and temperature difference become larger, the results predicted by the Hi-Z and simulation deviate from each other where the deviation increases with the increment of hot side temperature and temperature difference. Such discrepancies could be caused by using the different temperature-dependent material properties and the difference of the temperature variation within the thermoelectric unit.

Fig. 10 also shows the variation for power output with the load resistance in the experimental test under different conditions of hot side temperature. In these three figures, the experimental curve is in good agreement with the other two curves. However, actual results of the experiment have a minor difference with simulation and $\mathrm{Hi}-\mathrm{Z}$ calculator, particularly at the higher hot side temperature. There are two main reasons which lead to such a discrepancy. Firstly, instrument errors and reading errors are inevitable in the test. Moreover, due to the thermal loss in the test, the actual heat transfer to the test prototype is not as much as the cartridge heaters supplied. When the temperature increase, the temperature difference between test rig and ambient becomes larger, thus the heat loss was exacerbated. In the Fig. 10 (c), the experiment data shows the obvious difference with the other two curves.

\subsection{Heat transfer filling factor}

In practice, the installation space for TEG system is highly restricted, which means delicate considerations of location and configuration should be taken into account. Especially, there is little room for such an auxiliary system in the existing passenger car platform. On the other hand, the whole TEG system needs to take in more heat from the exhaust gas in the same area or length for higher recuperation performance. As the various designs of heat recovery system, the area of the heat exchanger in each TEG system are significantly different. In this present study, different topological design TEG systems from Purdue University is introduced here as a comparison [26, 27]. Provided that TEG systems can be easily scalable, we consider only the ratio of TEM and heat exchanger areas, rather than their absolute values. In this study, a new measurable indicator is described. The ratio of TEM to heat exchanger areas is named as the 
heat transfer filling factor: $f=A_{\text {TEM }} / A_{\text {Exchanger }}$. The heat transfer filling factor of different topological designed TEG systems and the CCTEG system are shown in Table 3.

Table 3 Heat transfer filling factor of different topological TEG

\begin{tabular}{cccccc}
\hline Topological Shape & Longitudinal & Transverse & Hexagonal & Cylindrical & $\begin{array}{c}\text { Concentric } \\
\text { Cylindrical }\end{array}$ \\
\hline Filling Factor & 0.458 & 0.43 & 0.521 & 0.575 & 0.655 \\
\hline
\end{tabular}

The heat transfer filling factor is a measurable indicator of the level of space utilisation in installed place. As seen in Table 3, the CCTEG system has the highest heat transfer filling factor which means, in the same space, more ATEM in the CCTEG system can be installed. Practically, the problem of narrow installation space can be solved for TEG system in existing vehicles without deep modifications. This factor can give guidance for further optimal design of TEG systems.

In most situations, the increment of back pressure in the whole exhaust system has an adverse effect on the fuel economy of the vehicle. By introducing the heat pipes, the CCTEG system has a higher heat transfer filling factor which means it can transport more heat in the same area. This will also help to reduce the complexity of the exhaust system and result in the decrease of back pressure increment. Furthermore, due to the high heat transfer filling factor, the same amount of coolant can obtain a better cooling effect. This benefit results in reducing the amount of power supply to the water pump, subsequently improving the fuel economy. Another advantage of applying heat pipes is that they may contribute to the obsolete of mufflers as the noise and vibrations in the exhaust can be absorbed in the heat pipe as protruded pin fins. This will be investigated as the integration of heat recovery and noise reduction technologies in the future.

\subsection{Influence of coolant parameter}

In addition to the effects of hot-side boundary condition and heat exchanger, the coolant flow rate also affects the maximum power output of the TEG system. Varying the coolant flow rate, the external resistances $\left(R_{L}\right)$ also change which leads to the different maximum power output of the whole system. At lower coolant flow rates, the overall internal temperature of the prototype is higher than that at higher flow rates because the temperature of the cold surfaces is greater at low flow rates. Therefore, given the fact that internal resistance increases with temperature, the matched load resistance for maximum power will increase.

In Fig. 11, the specific temperature differences of TEG modules at each specific coolant flow rate are illustrated with various hot side temperature conditions. From the figure, the temperature difference increases as the cooling flow rate increases can be observed. Additionally, the slopes of each plot that the highest rate of temperature difference increase occurred at the lower flow rates. As the flow rate increases, the slope ratio of temperature difference decreases accordingly. Moreover, the overall rate of temperature difference increase is larger at lower hot-side temperatures than that observed at higher temperatures. This increase can be attributed to the larger initial temperature difference applied between the hot and cold 
surfaces. As the flow rate and the heat flux are increased, the transient temperature difference between the surfaces decreases resulting in a lower rate of heat transfer.

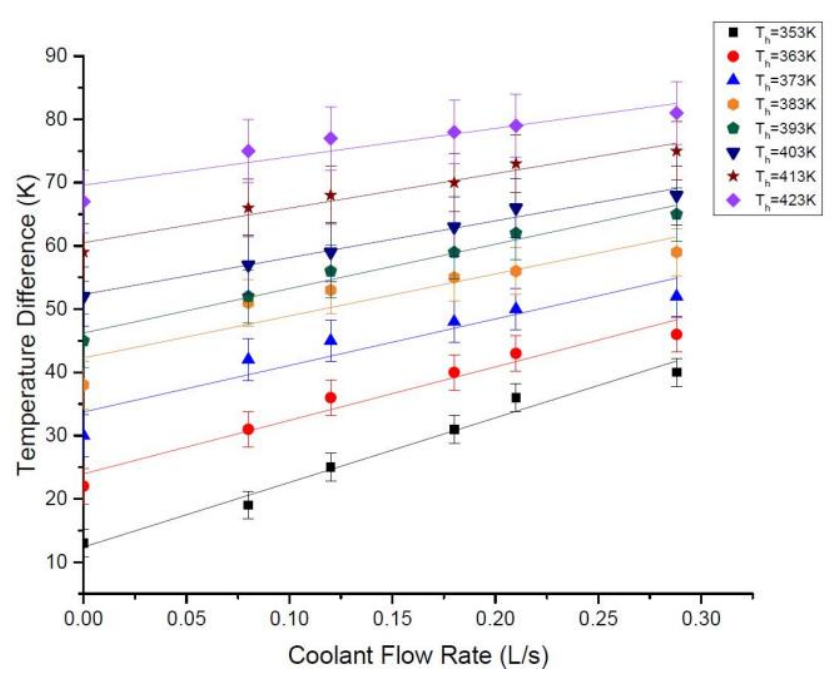

Fig. 11. Temperature difference under different coolant flow rate

\section{Conclusions}

This paper has presented the novel designs of a concentric cylindrical thermoelectric generator (CCTEG) and an annular thermoelectric module (ATEM). The study mainly aimed at accommodating more TEMs in the same limited space and to obtain more heat from the exhaust gas under the same engine working condition.

In order to verify this new concept, simulation studies about ATEM and square thermoelectric module (STEM) have been carried out. Besides, a module-scale prototype of CCTEG has been set up in the laboratory to investigate the performance and feasibility of this design. The experimental results have been compared with those of the simulation and Hi-Z performance calculator. The results show that ATEM plays a more significant role in heat transfer than original STEM due to its larger surface area. On account of the simulation and experimental results, the new model of CCTEG generates the same open circuit voltage and power output as the conventional thermoelectric generators. Moreover, the introduction of the heat pipe is a promising technology to enhance the radial direction heat transfer. By using the novel design of CCTEG in the vehicular exhaust system, the system can generate more power in the same length of exhaust pipe, thus can improve the fuel economy more significantly. Notably, a new index termed as the heat transfer filling factor $f$ has been introduced that quantities the level of space utilisation by TEMs where they are installed. This index can give guidance for further optimisation design of TEG systems.

Further studies on optimal design should be accomplished to improve the thermal conductivity of the whole system and optimise the geometry design of TEM. In order to investigate the performance under different working conditions, more experimental studies for CCTEG need to be carried out as well. These results could help to optimise the concentric CCTEG system to adapt to variable working conditions of vehicles. 


\section{Acknowledgements}

The work was supported by FAW R\&D Centre China and also partially by Ningbo Science and Technology Bureau's Technology Innovation Team Project under Grant No. 2016B10010. The authors would further acknowledge the support from China Scholarship Council (CSC) for the first author's scholarship.

\section{References}

[1] Ssennoga Twaha, Jie Zhu, Yuying Yan, Bo Li, A comprehensive review of thermoelectric technology: Materials, applicatio6ns, modelling and performance improvement, Renewable and Sustainable Energy Reviews, 65 (2016) 698-726.

[2] M. Karvonen, R. Kapoor, A. Uusitalo, V. Ojanen, Technology competition in the internal combustion engine waste heat recovery: a patent landscape analysis, Journal of Cleaner Production, 112 (2016) 3735-3743.

[3] D.V. Singh, E. Pedersen, A review of waste heat recovery technologies for maritime applications, Energy Conversion and Management, 111 (2016) 315-328.

[4] B.D. In, K.H. Lee, A study of a thermoelectric generator applied to a diesel engine, Proceedings of the Institution of Mechanical Engineers Part D-Journal of Automobile Engineering, 230 (2016) 133-143.

[5] D.K. Aswal, R. Basu, A. Singh, Key issues in development of thermoelectric power generators: High figureof-merit materials and their highly conducting interfaces with metallic interconnects, Energy Conversion and Management, 114 (2016) 50-67.

[6] T.J. Hendricks, S. Yee, S. Leblanc, Cost Scaling of a Real-World Exhaust Waste Heat Recovery Thermoelectric Generator: A Deeper Dive, Journal of Electronic Materials, 45 (2016) 1751-1761.

[7] H.P. Martin, A. Ponicke, M. Kluge, I. Sichert, A. Rost, S. Conze, K. Watzig, J. Schilm, A. Michaelis, TiOxBased Thermoelectric Modules: Manufacturing, Properties, and Operational Behavior, Journal of Electronic Materials, 45 (2016) 1570-1575.

[8] X. Liu, Y.D. Deng, K. Zhang, M. Xu, Y. Xu, C.Q. Su, Experiments and simulations on heat exchangers in thermoelectric generator for automotive application, Applied Thermal Engineering, 71 (2014) 364-370.

[9] C. Tao, G. Chen, Y. Mu, L.S. Liu, P.C. Zhai, Simulation and Design of Vehicle Exhaust Power Generation Systems: The Interaction Between the Heat Exchanger and the Thermoelectric Modules, Journal of Electronic Materials, 44 (2015) 1822-1833.

[10] H. Lee, Optimal design of thermoelectric devices with dimensional analysis, Applied Energy, 106 (2013) 7988.

[11] C.X. Liu, X.X. Pan, X.F. Zheng, Y.Y. Yan, W.Z. Li, An experimental study of a novel prototype for twostage thermoelectric generator from vehicle exhaust, Journal of the Energy Institute, 89 (2016) 271-281.

[12] X.Y. Liang, X.X. Sun, H. Tian, G.Q. Shu, Y.S. Wang, X. Wang, Comparison and parameter optimization of a two-stage thermoelectric generator using high temperature exhaust of internal combustion engine, Applied Energy, 130 (2014) 190-199.

[13] S.H. Yu, Q. Du, H. Diao, G.Q. Shu, K. Jiao, Effect of vehicle driving conditions on the performance of thermoelectric generator, Energy Conversion and Management, 96 (2015) 363-376.

[14] W. He, S.X. Wang, C. Lu, X. Zhang, Y.Z. Li, Influence of different cooling methods on thermoelectric performance of an engine exhaust gas waste heat recovery system, Applied Energy, 162 (2016) 1251-1258.

[15] X.Z. Wang, B. Li, Y.Y. Yan, S. Liu, J. Li, A study on heat transfer enhancement in the radial direction of gas flow for thermoelectric power generation, Applied Thermal Engineering, 102 (2016) 176-183.

[16] Y. Li, J.B. He, H.F. He, Y.Y. Yan, Z.X. Zeng, B. Li, Investigation of ultra-thin flattened heat pipes with sintered wick structure, Applied Thermal Engineering, 86 (2015) 106-118.

[17] Y. Li, Z.X. Li, C.Y. Chen, Y.Y. Yan, Z.X. Zeng, B. Li, Thermal responses of heat pipes with different wick structures under variable centrifugal accelerations, Applied Thermal Engineering, 96 (2016) 352-363.

[18] T. Zhang, Effects of Temperature-Dependent Material Properties on Temperature Variation in a Thermoelement, Journal of Electronic Materials, 44 (2015) 3612-3620.

[19] T. Zhang, Design and optimization considerations for thermoelectric devices, Energy Conversion and Management, 112 (2016) 404-412.

[20] Z.Q. Niu, H. Diao, S.H. Yu, K. Jiao, Q. Du, G.Q. Shu, Investigation and design optimization of exhaustbased thermoelectric generator system for internal combustion engine, Energy Conversion and Management, 85 (2014) 85-101.

[21] H.S. Lee, Thermal design: heat sinks, thermoelectrics, heat pipes, compact heat exchangers, and solar cells, John Wiley \& Sons, 2010.

[22] H. Scherrer, S. Scherrer, D. Rowe, Thermoelectric Handbook-Macro to Nano, ed. Rowe, DM, Taylor \& Francis, (2006). 
[23] L.L. Baranowski, G.J. Snyder, E.S. Toberer, Effective thermal conductivity in thermoelectric materials, Journal of Applied Physics, 113 (2013).

[24] S.Y. Zhou, B.G. Sammakia, B. White, P. Borgesen, Multiscale modeling of thermoelectric generators for the optimized conversion performance, International Journal of Heat and Mass Transfer, 62 (2013) 435-444.

[25] J.L. Gao, Q.G. Du, M. Chen, B. Li, D.W. Zhang, Assessing the accuracy of mathematical models used in thermoelectric simulation: Thermal influence of insulated air zone and radiation heat, Applied Thermal Engineering, 82 (2015) 162-169.

[26] S. Kumar, S.D. Heister, X.F. Xu, J.R. Salvador, G.P. Meisner, Thermoelectric Generators for Automotive Waste Heat Recovery Systems Part I: Numerical Modeling and Baseline Model Analysis, Journal of Electronic Materials, 42 (2013) 665-674.

[27] S. Kumar, S.D. Heister, X.F. Xu, J.R. Salvador, G.P. Meisner, Thermoelectric Generators for Automotive Waste Heat Recovery Systems Part II: Parametric Evaluation and Topological Studies, Journal of Electronic Materials, 42 (2013) 944-955.aa 